\title{
Structure in the winds of O-Type stars: observations and inferences
}

\author{
Alex W. Fullerton \\ Space Telescope Science Institute, 3700 San Martin Drive, Baltimore, MD 21218, USA \\ Herzberg Institute of Astrophysics, 5071 West Saanich Road, Victoria, BC V9E 2E7, Canada \\ email: fullerton@stsci.edu
}

\begin{abstract}
This review describes the observational evidence for structure in the winds of Otype stars due to large-scale perturbations and small-scale inhomogeneities. Despite considerable progress, a comprehensive theoretical framework that explains the origin. properties, and coexistence of wind structure on different spatial scales has yet to be constructed and incorporated into model atmosphere analyses. Consequently, it is not yet possible to assess the effect of non-stationary structures on different wind diagnostics in a rigorous way, with the result that accurate empirical determinations of mass-loss rates remain elusive.
\end{abstract}

Keywords. stars: early-type, stars: winds, outflows, stars: mass loss, ultraviolet: stars

\section{Introduction}

Modern understanding of continuous mass loss from early-type stars is based on the elegant theory of line-driven stellar winds that was introduced by Lucy \& Solomon (1970), developed by Castor, Abbott, \& Klein (1975), and refined by, e.g., Pauldrach, Puls, \& Kudritzki (1986; see also subsequent papers II - XV in this series). In this theory, the momentum required to accelerate material outward is transferred from the intense radiation field via scattering in many spectral lines. The standard model envisages the outflow as being (a) spherically symmetric; (b) smooth, with a monotonically increasing velocity; and (c) stationary. Our confidence in the standard model stems from its ability to explain the observational diagnostics of these outflows: P Cygni profiles; optical emission lines; and excess continuum emission at infrared and radio wavelengths. By any measure, the standard model of line-driven winds provides a rich harvest of physical insight and predictive power.

However, there are many indications that the winds of O-type stars contain velocity and density structure beyond that envisaged by the standard model. Some key observational manifestations of additional structure are illustrated in Fig. 1, which shows Copernicus spectra of the N v resonance doublet for $\zeta$ Ophiuchi (top) and $\zeta$ Puppis (bottom). Inset panels provide schematic comparisons of the expected wind-profile morphology for weak $(\zeta \mathrm{Oph})$ and saturated lines ( $\zeta$ Pup). Neither of the predictions reproduce the shape of the observed line profile. These failures point to the existence of wind structure on large and small spatial scales that is not incorporated in the standard model.

\section{Diagnostics of Large-Scale Wind Structure}

\subsection{Discrete Absorption Components}

Snapshot observations of unsaturated P Cygni profiles of UV resonance lines obtained with Copernicus and IUE showed that narrow, localized optical depth enhancements near the blue edge of the absorption trough are common among the $\mathrm{O}$ stars; see the 

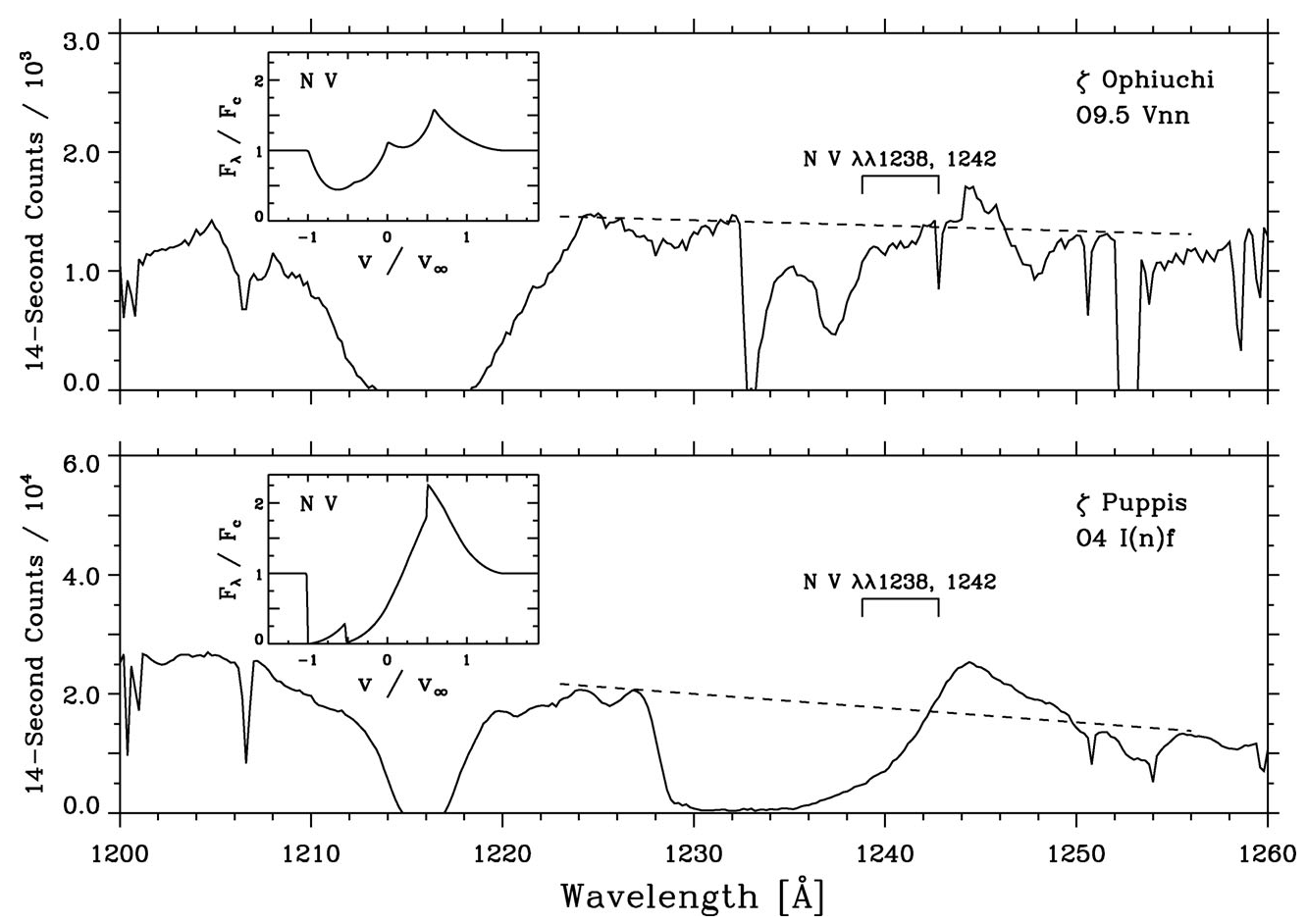

Figure 1. Copernicus spectra of the Nv $\lambda \lambda 1238,1242$ resonance doublet of $\zeta$ Oph (top) and $\zeta$ Pup (bottom). Inset panels show predictions from the standard model. Inspired by Fig. 1 of Morton (1976).

$\mathrm{Nv}$ profiles of $\zeta \mathrm{Oph}$ in Fig. 1. From a large survey of 203 Galactic O stars observed with IUE, Howarth \& Prinja (1989) estimated that $~ 97 \%$ of all O stars exhibit "narrow absorption components" (NACs) at any one time. Such a high incidence implies that the phenomenon is essentially universal.

The presence of NACs implies the existence of additional large-scale wind structure beyond that predicted by the standard model. Consider that the Sobolev optical depth of the wind in the radial direction is

$$
\tau_{\text {rad }}(r) \propto q_{i}(r) \rho(r)\left(\frac{\mathrm{d} v}{\mathrm{~d} r}\right)^{-1}
$$

where $q_{i}$ is the local ion fraction of the species responsible a particular wind line; $\rho$ is the local density; and $\mathrm{d} v / \mathrm{d} r$ is the local velocity gradient. Since the NACs represent enhanced optical depth, at least one of these parameters must be different from its standard value: either the ion fraction is increased, the density is increased, or the velocity gradient is shallower at the location of the NAC; or all three changes are combined, since the variables are not independent. Furthermore, since the NACs are often very deep (see Fig. 1), the absorbing gas responsible for them must be optically thick and cover a substantial fraction of the stellar disk. Thus, we infer that the NACs are due to changes in the velocity field, density, or ionization structure of the smooth wind that are localized in velocity, but occur on spatial scales that are comparable to the stellar radius.

Since NACs are always present in unsaturated wind profiles of a given star, they were initially interpreted in terms of persistent structures in the wind; see, e.g., Lamers et al. (1982). However, further analysis of sporadic time series (see, e.g., Prinja \& Howarth 1986) showed that NACs vary on time scales of a day or less. Intensive monitoring with 

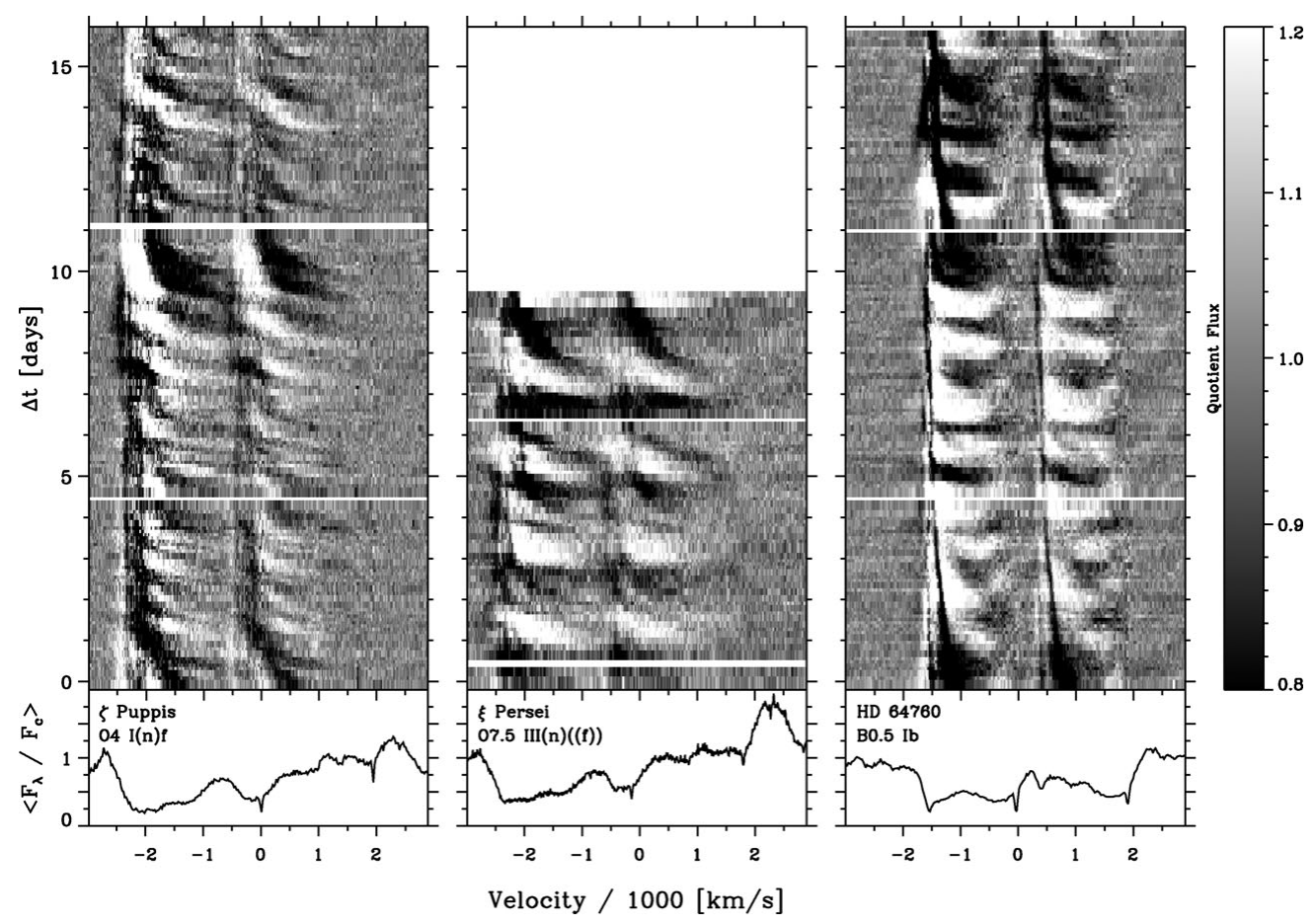

Figure 2. Spectroscopic time series observations of the Si IV $\lambda \lambda$ 1394, 1403 resonance doublet of $\zeta$ Pup (left). $\xi$ Per (middle), and HD 64760 (right). The time series of $\zeta$ Pup and HD 64760 were obtained during the IUE "MEGA Campaign", while the observations of $\xi$ Per were obtained with $I U E$ in 1994 October. In all cases the dynamic spectra have been normalized by the mean spectrum from the time series.

IUE of $\xi$ Persei [O7.5 III(n)((f)); Prinja et al. 1987] and 68 Cygni [O7.5 III:n((f)); Prinja \& Howarth 1988] demonstrated the fundamentally dynamic and repetitive nature of the phenomenon. As illustrated in Fig. 2 for three key objects, broad, weak absorptions are initially detected at quite small velocities. These absorptions narrow and strengthen as they accelerate blueward through the absorption trough, until they eventually merge with the persistent, high-velocity NAC. Consequently, the NACs are now recognized as the end products of the more general phenomenon of "discrete absorption components" (DACs), which move through a large swath of the absorption trough. With this understanding, we infer from the ubiquity of NACs that DACs must also be universally present in unsaturated wind lines of $\mathrm{O}$ stars.

Although comparatively few stars have been observed intensively, the following general properties have been identified by tenacious observational effort (e.g., Kaper et al. 1996; Prinja et al. 2002): (a) DACs accelerate slowly through the absorption trough compared to the expectations based on the mean flow of the wind; (b) DACs recur on intervals related to the estimated stellar rotation period $\left(P_{\text {rot }}\right)$; and $(c)$ although DACs are always present in multi-epoch observations of the same star and the pattern of variability is always similar, the variations do not seem to be phase locked over intervals of several months.

As a direct test of the connection between DAC activity and the rotation period of the underlying star, $\zeta$ Pup and HD 64760 were monitored for $\sim 16$ consecutive days in 1995 January as part of the IUE "MEGA Campaign" (Massa et al. 1995). These exquisite time series are shown in Fig. 2 along with an equally heroic effort on $\xi$ Per that 

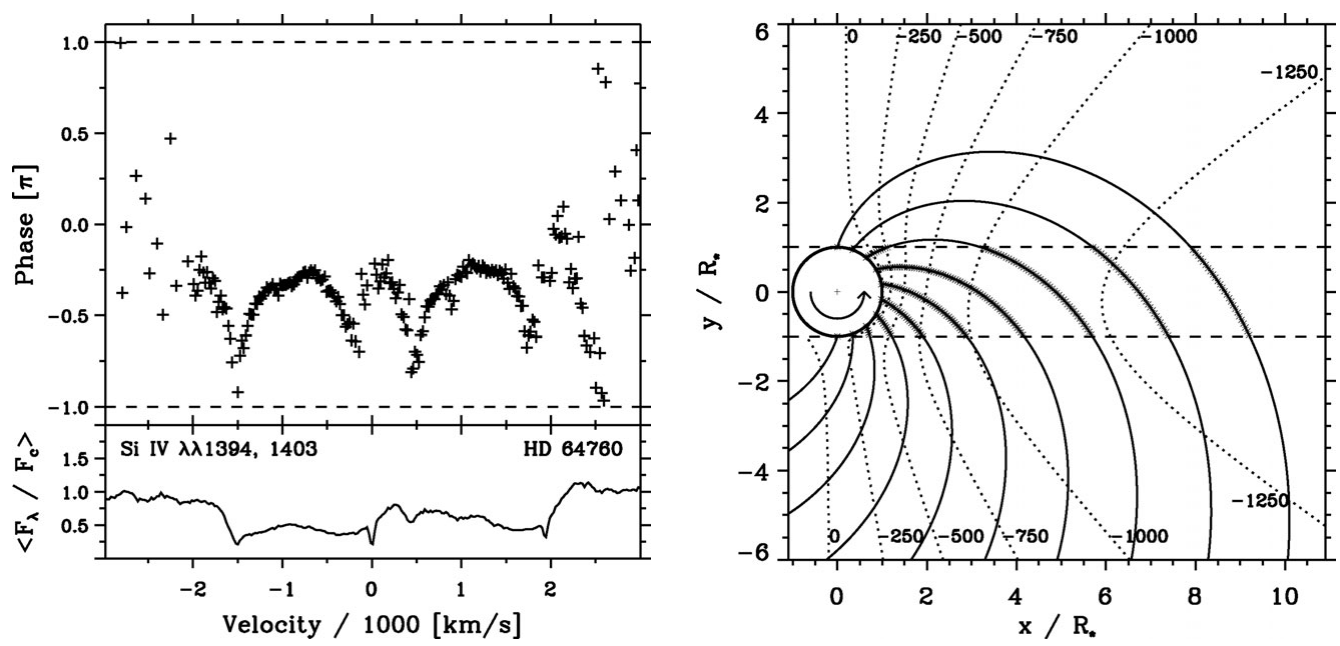

Figure 3. Left: Phase of the 1.2-day PAM as a function of position in the absorption trough of the Si IV doublet of HD 64760. Right: Schematic illustration of the origin of phase bowing from Fullerton et al. (1997).

included co-ordinated ground-based observations (de Jong et al. 2001). The duration of the observations covered 3-4 consecutive repetitions of the estimated $P_{\text {rot }}$ for each star; and in all cases, variation related to this period were detected. However, each star is a little different. For $\zeta$ Pup, the DACs recur every 0.80 days, and the entire profile is modulated every $\sim 5.2$ days $\sim P_{\text {rot }}$ (Howarth et al. 1995) while for $\xi$ Per the DACs recur every 2.086 days $\simeq P_{\text {rot }} / 2$ (de Jong et al. 2001). Curiously, DACs of HD 64760 recur only once, which indicates that their recurrence time is longer than $P_{\text {rot }}$. However, a strikingly different type of variation is immediately apparent in Fig. 2 in the form of periodic absorption modulations.

\subsection{Periodic Absorption Modulations}

The periodic component of wind variability exhibited by HD 64760 is visible in Fig. 2 as light and dark bands that recur every 1.2 days (Fullerton et al. 1997). These variations are due to modulations of the optical depth in the absorption trough, not enhancements. These periodic absorption modulations (PAMs) do not move sequentially through the absorption trough, but instead affect a broad range of velocities nearly simultaneously. Consequently, PAMs are a distinctly different phenomenon from DACs, though obviously they can coexist.

Although the PAMs of HD 64760 are thus far unique, they provide many insights into the geometry of repetitive wind structures through the phenomenon of "phase bowing". Phase bowing can be seen especially well in Fig. 2 near $\Delta \mathrm{T}=2$ days, where curved, upward-facing bright features corresponding to a single phase of the modulation evolve simultaneously toward higher and lower velocities in the absorption trough. It is even more evident in the distribution of the sinusoidal phase constant associated with the modulations as a function of position in the absorption trough (Fig. 3), which shows a maximum at an intermediate velocity. Owocki et al. (1995) explained this behavior in terms of the way that an azimuthally extended structure exits the line of sight to a distant observer. As shown schematically in Fig. 3, such structure first exits the column of material projected against the disk of the star at an intermediate velocity, and thereafter exits at both large and small line-of-sight velocities simultaneously, as observed. 
Thus, phase bowing is now believed to be a robust diagnostic of the presence of longitudinally extended - probably spiral-shaped - structures in the wind. This geometry is not uniquely associated with PAMs. For example, by carefully comparing the behaviour of the phase constant in different wind lines, de Jong et al. (2001) detected phase bowing associated the DACs of $\xi$ Per.

\subsection{Co-Rotating Interaction Regions}

Following the suggestion of Mullan (1984), cyclical variability in hot-star winds is now attributed to the behaviour of azimuthally extended perturbations known as co-rotating interaction regions (CIRs). CIRs are a well known phenomenon in the solar wind that are caused by differences in the initiation of the wind at different locations. These changes in boundary conditions cause "fast" and "slow" streams to emerge from adjacent regions of the stellar surface along nearly radial trajectories. However, as the star rotates, the "fast" wind from one sector overtakes the "slow" wind that previously emerged from an adjacent sector. The resultant interaction produces a shock and a shallow, plateau-like velocity gradient. Although material continues to flow through the interaction zone, its slower speed also causes a localized density enhancement (compared with an unstructured wind). The net result is a long-lived, spiral-shaped structure in the wind that rotates with a period determined by the surface phenomenon responsible for creating the "fast" and "slow" wind streams in the first place.

CIRs provide a natural explanation for essentially all the properties of cyclical wind variability; see, e.g., the hydrodynamic simulations of Cranmer \& Owocki (1996), Dessart (2004), and Lobel \& Blomme (2008). Enhanced optical depth is readily produced by the velocity plateau and, secondarily, the density enhancement caused by the interaction region. The systematic narrowing of the DAC as it propagates blueward through the absorption trough is accounted for by projection effects along the spiral-shaped CIR as it rotates into the line of sight, while "phase bowing" can be attributed to the behaviour of the spiral as it exits the near hemisphere of the wind. Most importantly, the slow apparent motion of the DAC does not imply that material is also moving much more slowly than expected, because the DAC is produced by different material flowing through the interaction region at different times. The apparent acceleration is determined by the shape of the CIR, which in turn is determined by the processes at the stellar surface that are responsible for the emergence of "fast" and "slow" wind streams. This coupling between the CIR and the stellar surface ultimately determines the recurrence period of cyclical wind variations in a particular star.

Thus, in the current paradigm, photospheric processes are ultimately responsible for large-scale wind variability. The ubiquity of DACs (or at least NACs) requires a ubiquitous "trigger" mechanism: i.e., a process or processes that organize the stellar surface into azimuthal sectors that alternately produce "fast" and "slow" outflows of material. Motivated by the expected temperature variations associated with nonradial pulsations (NRP) of modest degree (see, e.g., Kaufer et al. 2006), hydrodynamic simulations use the artifice of one or more bright spots distributed around the star to produce these streams. Of the "usual suspects" - pulsations or magnetic fields - it does seem more likely that multi-mode pulsation can provide the necessary coverage of the parameter space occupied by O- and B-type stars, if indeed a single "trigger" is responsible for creating CIRs.

Lobel \& Blomme (2008) also show that retrograde NRP can lead to recurrence times that are substantially longer than the rotation period of the star. For the particular case of HD 64760 (Fig. 2), they show that the long interval between the recurrence of DACs can be accommodated. Thus, it may be that the interplay between the pattern speed of multimode NRP and the rotation of the stellar surface prevent DACs from being 
phase-locked over long intervals, even though the patterns are similar in multi-epoch observations (see, e.g., Kaper et al. 1999). In the specific case of HD 64760, the relationship between the DACs and the PAMs needs clarification, since it is not obvious how multiple instances of CIRs with extremely different time scales can coexist in the wind.

\section{Diagnostics of Small-Scale Wind Structure}

In addition to the large-scale spiral structures implied by the cyclical variability of wind lines, there is compelling evidence that the winds of O-type stars are inhomogeneous over much smaller distances.

\subsection{Transient Emission-Line Substructures}

The most direct indication of small-scale density enhancements in stellar winds comes from observations of narrow, transient substructures in strong emission lines (e.g, $\mathrm{H} \alpha$, He II $\lambda 4686)$. These lines are predominantly formed by recombination in the wind, which is a two-body or "density squared" (hereafter $\rho^{2}$ ) process. Consequently, the narrow bumps of excess emission trace regions of enhanced density that are localized in velocity (which probably implies being localized in space, too). The bumps move systematically away from line center over the course of a few hours, which is broadly consistent with the flow time and suggests that the inhomogeneities are carried along by the wind. Similar transients have been observed extensively in the much broader emission lines formed in the winds of Wolf-Rayet stars; see, e.g., Moffat et al. (1988). However, it is quite difficult to detect transient features in the narrower and weaker emission profiles of O-stars and to date they have only been observed in the wind profiles of $\zeta$ Pup (O4 I(n)f; Eversberg et al. 1998, Lépine \& Moffat 2008) and HD 93129A (O2 If*; Lépine \& Moffat 2008).

\subsection{Black Troughs of Saturated UV Resonance Lines}

Strongly saturated resonance lines of $\mathrm{O}$ supergiants typically exhibit extended intervals of zero residual flux; see, e.g., the $\mathrm{NV}$ resonance doublet of $\zeta$ Pup in Fig. 1. These "black troughs" are impossible to understand in the context of the standard model, which predicts that a saturated P Cygni profile will have no residual flux only at its blue edge, because all other wavelengths have a component of emission directed toward a distant observer. Lucy (1982) recognized that "black troughs" are caused by scattering in a wind with a multiply non-monotonic velocity field, which leads to enhanced backscattering and a systematic reduction in the blue-shifted (forward-scattered) emission; see Fig. 4. Thus, "black troughs" are a signature of non-monotonic velocity structure in a stellar wind, which is presumably accompanied by localized density inhomogeneities. As discussed by Puls et al. (1993), a substantial amount of small-scale velocity structure is required to get extremely black profiles; see also the discussion by Sundqvist et al. (2010).

\subsection{Soft Blue Edges of UV Resonance Lines}

A second morphological peculiarity of the absorption troughs of $\mathrm{P}$ Cygni profiles is that their blue edges do not rise to the continuum as steeply as expected. This "softness" of the blue edge is particularly evident in saturated lines, but is commonly seen in weaker lines too; see Fig. 1. In the standard model, the most blue-shifted absorption in a strong $\mathrm{P}$ Cygni profile denotes the terminal velocity of the wind $\left(v_{\infty}\right)$, which is the largest velocity along the line of sight to the star (provided that the ion responsible for the resonance transition exists throughout the wind). Since, by definition, no wind material exists at larger velocities to scatter light from the star, the transition to the local stellar 

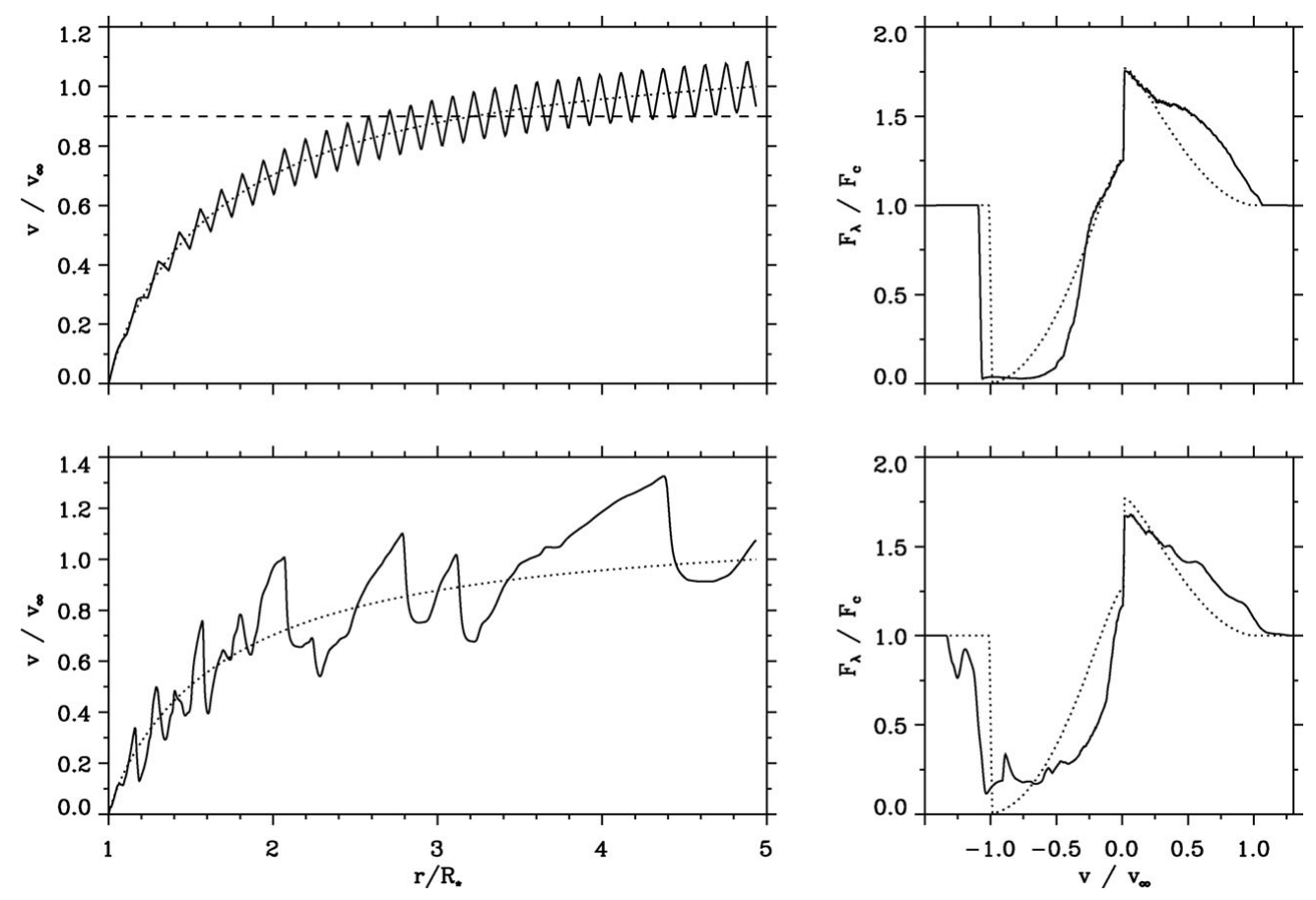

Figure 4. Upper: Schematic illustration of the creation of a black trough from multiple scattering interactions in a non-monotonic velocity field. In the left-hand panel, a sawtooth variation is imposed on the smooth, monotonic velocity profile (dotted). The dashed horizontal line indicates that a photon interacts with the sawtooth profile many times. The right-hand panel shows that preferential back-scattering in the structured wind creates extended black troughs, which are not completely black due to "vorosity". Since structure occurs in spherical shells for this 1D simulation, excess red-shifted emission is seen from back-scattering in the far hemisphere of the wind. Lower: Velocity structure from a 1-D radiation hydrodynamics simulation of the line-deshadowing instability. In this case, there are not enough interactions between a continuum photon and the wind to backscatter all the flux and the profile is not saturated. Adapted from Puls et al. (1993).

continuum must necessarily be abrupt. Instead, the observed "softness" of the blue edge indicates that there is a distribution of material at velocities in excess of the terminal velocity; i.e., there is a dispersion of velocities about the mean velocity field. This velocity dispersion also implies localized variations in density within the wind.

\subsection{Discrepant Mass-Loss Estimates}

Large, systematic differences in estimates of the mass-loss rate $(\dot{M})$ from diagnostics with different density dependencies provide the least direct evidence for small-scale structure, but constitute the strongest indication that such structure is a universal property of O-star winds. The key distinction is between (a) scattering of continuum photons by UV resonance lines, which is linearly proportional to density $(\rho)$; and (b) emission processes (recombination lines, free-free continuum emission), which are proportional to $\rho^{2}$. However, only $\dot{M} q_{i}$ can be determined from wind profiles of unsaturated UV resonance lines. Reliable values of $q_{i}$ are notoriously difficult to estimate theoretically or measure empirically for the most commonly observed species, which means that $\dot{M}$ is poorly constrained. As a result, recent estimates of $\dot{M}$ for O-type stars have all relied on $\rho^{2}$ diagnostics, especially $\mathrm{H} \alpha$. 


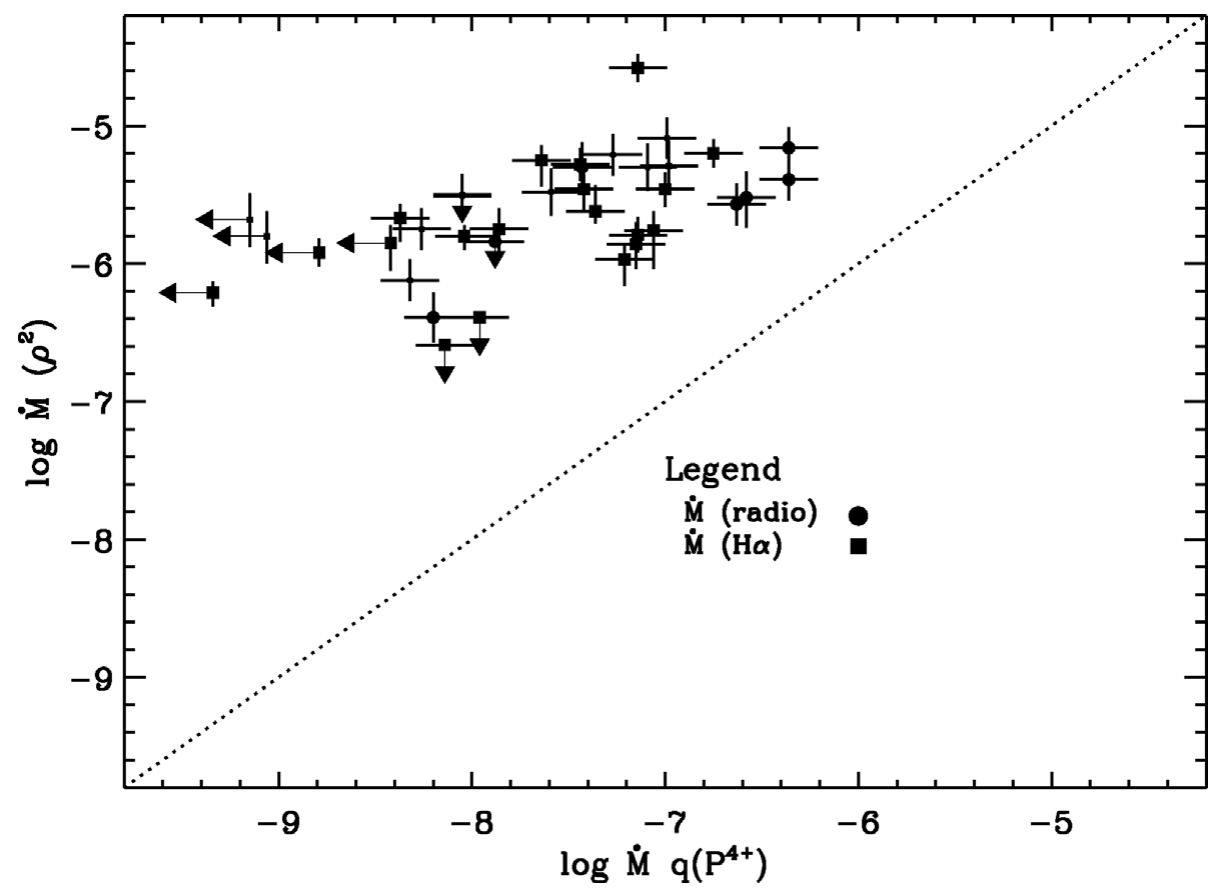

Figure 5. Comparison of $\dot{M}$ determined from $\rho^{2}$ diagnostics and the $\mathrm{P}$ V resonance lines of 40 Galactic O-type stars. Stars with temperature class between O4 and O7.5 are closest to the 1-1 correlation line, but have discrepant estimates of $\dot{M}$ by a factor of 20. From Fullerton, Massa, \& Prinja (2006).

The launch of FUSE in 1999 changed the situation dramatically by providing access to the rich suite of resonance-line diagnostic between $\sim 900$ and $1200 \AA$. For O-type stars, the $\mathrm{P} V \lambda \lambda 1122,1128$ doublet is crucially important, for two reasons. First, since $\mathrm{P}$ is not a cosmically abundant element, the absorption troughs of the P V P Cygni profiles are never saturated and $\dot{M} q_{i}$ can be measured accurately. Second, state-of-the-art model atmosphere calculations based on the standard model predict that $P^{4+}$ is the dominant ion in the winds of mid- to late- O-type stars. For these stars, $q_{i}$ is known a priori to lie between $\sim 0.5$ and 1 . Consequently, simple analysis of $\mathrm{PV}$ wind profiles should yield measurements of $\dot{M}$ that agree with determinations from $\rho^{2}$ diagnostics to within a factor of $\sim 2$ for at least some $\mathrm{O}$ stars.

However, Fig. 5 shows that measurements of $\dot{M}$ from $\rho^{2}$ diagnostics for a "blue-ribbon" sample of 40 Galactic O-type stars are always greater than measurements from the $\mathrm{PV}$ resonance lines by a factor of at least 20 (Fullerton, Massa, \& Prinja 2006). Detailed model atmosphere calculations indicate similar levels of disagreement between $\mathrm{H} \alpha$ and the $\mathrm{P} \mathrm{V}$ doublet for O-type supergiants in the Galaxy (Bouret et al. 2005), Large Magellanic Cloud (Crowther et al. 2002; Massa et al. 2003) and Small Magellanic Cloud (Hillier et al. 2003).

The simplest resolution of this "P $\mathrm{V}$ problem" is to assume that hot-star winds are significantly "clumped" on small scales, in which case $\rho^{2}$ diagnostics necessarily over estimate the amount of material in the wind when they are interpreted within the context of the standard model. Thus, we infer from the discrepancy illustrated in Fig. 5 that O-star winds are inhomogeneous on small spatial scales; and, as a corollary, that previous estimates of $\dot{M}$ are too large. Strictly speaking, the discrepancy only exists for the subset of $\mathrm{O}$ stars for which $\mathrm{P}^{4+}$ is the dominant ion. However, there is no reason to suppose 
that the mechanism responsible for clumping the wind is limited to this subset, so the inference is more generally valid.

\subsection{The Origin and Nature of Clumping}

The strong line-deshadowing instability (LDI) is an intrinsic property of the line-driving mechanism (Owocki \& Rybicki 1984). As such, it provides a unifying framework to interpret the observational signatures of small-scale structure. In particular, numerical simulations of the nonlinear growth of the LDI by Owocki, Castor, \& Rybicki (1988) and Feldmeier (1995) show that small amounts of material are accelerated to velocities in excess of $v_{\infty}$, exactly as required to explain the "soft" blue edges of P Cygni profiles. In 1D simulations, subsequent interactions between this fast moving gas and slower material at larger radii compress the wind into a series of dense shells, which necessarily make the velocity profile non-monotonic (black troughs; Fig. 4). Exploratory simulations in 2D by Dessart \& Owocki (2005) suggest that these shells fragment laterally into many small clumps, which manifest themselves as transient emission-line substructures and significantly bias determinations of $\dot{M}$. Shocks and collisions between dense shells also produce X-rays distributed throughout the wind; see, e.g., the article by Cohen \& Leutenegger in these proceedings.

Although the LDI provides a firm theoretical basis to understand the origin of smallscale structure, there are still many uncertainties concerning the size and shape of the clumps. Fortunately, work to reconcile estimates of $\dot{M}$ from different diagnostics provides some general constraints on these properties. Initial attempts to incorporate clumping in model atmosphere calculations relied on a two-component model, in which the wind was assumed to consist of (a) optically thin clumps characterized by enhanced density compared to predictions of the standard model; and (b) vacuum. Consistent estimates of $\dot{M}$ can be obtained with this approach, essentially by changing the prevailing ionization equilibrium of an element (e.g., P) in favor of lower ionization stages in denser environments. Very small volume filling factors are derived for the clumps in some cases (e.g., Bouret et al. 2005), which implies that the wind is mostly vacuum and that the clumps are very dense.

However, this approach does not adequately treat radiation transfer in spectral lines because it does not contain any length scales. Consequently, there is no way of testing whether the clumps are optically thick, which they must be for some of the implied overdensities. The character of the radiation transfer in the wind changes once the clumps become optically thick, because (a) radiation is trapped within the clumps; and (b) channels open up between the clumps that permit radiation from the star to leak out; i.e., the wind becomes "porous". The leakage implied by porosity reduces the effective optical depth of the wind (Owocki \& Cohen 2006; Oskinova et al. 2007) in a particular transition. Oskinova et al. (2007) recognized that resonance transitions become optically thick before other transitions, and consequently a clumped wind could be a porous medium for the $\mathrm{P} v$ doublet but not for $\mathrm{H} \alpha$. By allowing continuum light to "leak" around clumps that are optically thick in resonance lines, Oskinova et al. (2007) obtained reasonable fits to both the $\mathrm{P} v$ doublet and the $\mathrm{H} \alpha$ profile of $\zeta$ Pup with only a modest reduction in the "smooth wind" value of $\dot{M}$.

Owocki (2008) realized that the non-monotonic velocity field produced by the LDI has gaps in the velocity field that continuum photons can also leak through. This "porosity in velocity space" (dubbed "vorosity" by Owocki) is distinct from porosity in physical space, but also reduces the effective optical depth of the wind in different lines. In an impressive exploratory study, Sundqvist et al. (2010) confirmed that both porosity (due to optically thick clumps) and vorosity (due to velocity gaps between clumps) play a 
similar role in reducing the effective optical depth of the wind in intrinsically strong transitions. By accounting for "leakage" in velocity and physical space, they showed that it is in principle possible to obtain concordance between different mass-loss diagnostics.

However, Sundqvist et al. (2010) could not obtain satisfactory agreement between different diagnostics using the wind structure predicted by the current generation of radiation-hydrodynamics simulations of the LDI. Compared with these models, it appears that (a) structure must be initiated deeper in the wind; and (b) clumps need a greater internal velocity gradient. Further work to simulate the time-dependent structure of linedriven winds is required to determine whether these shortcomings are fundamental or simply artifacts of the current generation of models. An interesting twist might be to incorporate deep-seated perturbations from shallow convection zones to trigger the onset of clumping nearer to the photosphere (Cantiello et al. 2009).

\section{What Does a O-Star Wind Look Like?}

How should we picture the wind of an O-type star? The idea of a smooth, spherically expanding outflow is well entrenched in our minds, and certainly provides a useful point of departure for thinking about O-star winds. However, both theory and observations have encouraged us to consider the wind as an ensemble of distinct clumps, each with its own size, velocity, and propensity to interact with each other and the low-density medium that surrounds them. At the same time, variability studies have convinced us that large-scale spiral waves are embedded in the outflow and that these structures are continually renewed by photospheric processes that control the emergence of the wind.

Since observations suggest that each of these pictures contains an element of truth, the current challenge is to synthesize them into a coherent physical theory. There are a variety of issues that must be resolved, but foremost is to demonstrate that largescale CIRs can coexist with the LDI. Initial attempts to include CIRs in time-dependent radiation hydrodynamics simulations by Owocki (1999) met with mixed success, with the survival of a CIR depending on the precise treatment of the nonlocal line force. At present, further progress on this fundamental issue appears to be limited by the numerical techniques required to treat this difficult, two-dimensional problem.

An observational challenge is to identify definitively the "trigger mechanism" responsible for CIRs. If, e.g., DACs are the observational manifestation of CIRs and CIRs are produced by temperature variations on the stellar surface due to NRP, then the ubiquity of DACs implies that all O stars should be non-radial pulsators. This is a testable prediction, which receives some support from the widespread occurrence of photospheric line profile variations (Fullerton et al. 1996), though the much sought connection between photospheric activity and DACs has proven difficult to demonstrate explicitly (see, e.g., de Jong et al. 2001). This difficulty is now compounded by the lack of long-term spectroscopic monitoring capability in space. Our community desperately needs access to a modern version of IUE to make continued progress!

Understanding the time-dependent structure of hot-star winds is a fascinating problem for observers and theoreticians alike. However, this research is also important in a broader astrophysical context, because we cannot properly quantify the biases associated with different $\dot{M}$ diagnostics until we have a clearer understanding of the origin and nature of wind structure on all spatial scales. Initial indications are that the changes in mass flux associated with large-scale structure in the form of CIRs are rather small, (at least for HD 64760; Lobel \& Blomme 2008), whereas changes in the interpretation of massloss diagnostics due to the presence of small-scale structure are large (factor of 5?) and very uncertain. Until a unified treatment of all the different forms of wind structure 
becomes available, the mass-loss rates of O-type stars will necessarily remain uncertain by unacceptably large factors.

\section{References}

Bouret, J.-C., Lanz, T., \& Hillier, D. J. 2005, A\&A, 438, 301

Cantiello, M., Langer, N., Brott, I., de Koter, A. et al. 2009, A\&A, 499, 279

Castor, J. I., Abbott, D. C., \& Klein, R. I. 1975, ApJ, 195, 157

Cranmer, S. R. \& Owocki, S. P. 1996, ApJ, 462, 469

Crowther, P. A., Hillier, D. J., Evans, C. J., Fullerton, A. W. et al. 2002, ApJ, 579, 774

de Jong, J. A., Henrichs, H. F., Kaper, L., Nichols, J. S. et al. 2001, A\&̈A, 368, 601

Dessart, L. 2004, A\& $A$ A, 423, 693

Dessart, L. \& Owocki, S. P. 2005, A\&A A, 437, 657

Eversberg, T., Lepine, S., \& Moffat, A. F. J. 1998, ApJ, 494, 799

Feldmeier, A. 1995, A\&SA, 299, 523

Fullerton, A. W., Gies, D. R., \& Bolton, C. T. 1996, ApJS, 103, 475

Fullerton, A. W., Massa, D. L., \& Prinja, R. K. 2006, ApJ, 637, 1025

Fullerton, A. W., Massa, D. L., Prinja, R. K., Owocki, S. P. et al. 1997, A\&A, 327, 699

Hillier, D. J., Lanz, T., Heap, S. R., Hubeny, I. et al. 2003, ApJ, 588, 1039

Howarth, I. D. \& Prinja, R. K. 1989, ApJS, 69, 527

Howarth, I. D., Prinja, R. K., \& Massa, D. 1995, ApJ (Letters), 452, L65

Kaper, L., Henrichs, H. F., Nichols, J. S., Snoek, L. C. et al. 1996, A\&AS, 116, 257

Kaper, L., Henrichs, H. F., Nichols, J. S., \& Telting, J. H. 1999, A\&A, 344, 231

Kaufer, A., Stahl, O., Prinja, R. K., \& Witherick, D. 2006, A\& $A, 447,325$

Lamers, H. J. G. L. M., Gathier, R., \& Snow, Jr., T. P. 1982, ApJ, 258, 186

Lépine, S. \& Moffat, A. F. J. 2008, $A J, 136,548$

Lobel, A. \& Blomme, R. 2008, ApJ, 678, 408

Lucy, L. B. 1982, ApJ, 255, 278

Lucy, L. B. \& Solomon, P. M. 1970, ApJ, 159, 879

Massa, D., Fullerton, A. W., Nichols, J. S., Owocki, S. P. et al. 1995, ApJ (Letters), 452, L53

Massa, D., Fullerton, A. W., Sonneborn, G., \& Hutchings, J. B. 2003, ApJ, 586, 996

Morton, D. C. 1976, ApJ, 203, 386

Moffat, A. F. J., Drissen, L., Lamontagne, R., \& Robert, C. 1988, ApJ, 334, 1038

Mullan, D. J. 1984, ApJ, 283, 303

Oskinova, L. M., Hamann, W.-R., \& Feldmeier, A. 2007, A\&A, 476, 1331

Owocki, S. P. 1999, in: B. Wolf, O. Stahl, \& A. W. Fullerton (eds.), IAU Colloq. 169: Variable and Non-spherical Stellar Winds in Luminous Hot Stars, Lecture Notes in Physics, Berlin Springer Verlag 523, p. 294

Owocki, S. P. 2008, in: W.-R. Hamann, A. Feldmeier, \& L. M. Oskinova (eds.), Clumping in Hot-Star Winds, p. 121

Owocki, S. P. \& Cohen, D. H. 2006, ApJ, 648, 565

Owocki, S. P. \& Rybicki, G. B. 1984, ApJ, 284, 337

Owocki, S. P., Castor, J. I., \& Rybicki, G. B. 1988, ApJ, 335, 914

Owocki, S. P., Cranmer, S. R., \& Fullerton, A. W. 1995, ApJ (Letters), 453, L37

Pauldrach, A., Puls, J., \& Kudritzki, R. P. 1986, A\&A, 164, 86

Prinja, R. K. \& Howarth, I. D. 1986, ApJS, 61, 357

Prinja, R. K. \& Howarth, I. D. 1988, MNRAS, 233, 123

Prinja, R. K., Howarth, I. D., \& Henrichs, H. F. 1987, ApJ, 317, 389

Prinja, R. K., Massa, D., \& Fullerton, A. W. 2002, AछA, 388, 587

Puls, J., Owocki, S. P. \& Fullerton, A. W. 1993, A\&A, 279, 457

Sundqvist, J. O., Puls, J., \& Feldmeier, A. 2010, A\&A, 510, A11 


\section{Discussion}

MEYNET: You showed a very impressive difference between the $\rho^{2}$ diagnostics and the $P^{4+}$ UV diagnostics for $\dot{M}$. Does this difference decrease (for instance) when the metallicity decreases?

FUlLERTON: Very similar discrepancies are seen in samples of O-type stars observed with FUSE in both the LMC (Crowther et al. 2002, Massa et al. 2003) and SMC (Hillier et al. 2003). Although these samples are limited, they don't provide any evidence to suggest that the degree of clumping depends strongly on the overall metallicity of the star.

Lobel: Thank you for this clear review. You made the point that it is difficult to reconcile large-scale structures in the wind with the small-scale structures. It appears to me that we should separate the large-scale wind structures that cause DACs from those that produce the rotational modulations. Could you comment on the "phase bowing" in the modulations, for example, observed in IUE spectra of HD 64760? Some of its modulations do not reveal the phase bowing and are almost completely flat. Instead of the large-scale wind spirals we need for DACs, the modulations therefore require almost 'spoke-like' density enhancements in wind regions of $r \leqslant 10 R_{\star}$. Hydrodynamic modelling has recently shown that the modulations can be caused by pressure waves at the surface, instead of the spots or brighter equatorial regions that cause the large-scale CIRs.

Fullerton: I'll look forward to learning more about your simulations that include perturbations from pressure waves! As for "phase bowing": I agree that some occurrences of the PAMs exhibit more curvature than others in dynamic spectra (e.g., Fig. 2); but I think that most of the PAMs are curved. My analysis of "phase bowing" is based on the distribution of the sinusoidal fitting constant as a function of position in the absorption trough shown in Fig. 3, which is admittedly less sensitive to any peculiarities associated with an individual occurrence of the modulation.

KHOLTYGin: What can you say about the small-scale structure of large-scale structures? Is it possible that large-scale streams is the stellar wind of OB stars consist of small clumps?

Fullerton: Very little is known about the structure of the CIRs from observations, but I think it is plausible that the "fast" and "slow" streams responsible for them could be composed of small blobs of material. One implication would be that the onset of clumping occurs very deep, which is not what simulations of the LDI currently predict.

MASSA: I would like to point out that the Cosmic Origins Spectrograph on HST can obtain spectra in the FUSE range with roughly the same sensitivity as FUSE and resolving power of $8,000-10,000$. Calibration observations are being obtained at this time. 